\title{
Monetary risk measures for stochastic processes via Orlicz duality
}

\author{
Christos E. Kountzakis ${ }^{1} \cdot$ Damiano Rossello $^{2}$ (D
}

Received: 5 March 2020 / Accepted: 14 May 2021 / Published online: 29 May 2021

(c) The Author(s) 2021

\begin{abstract}
In this article, we extend the framework of monetary risk measures for stochastic processes to account for heavy tailed distributions of random cash flows evolving over a fixed trading horizon. To this end, we transfer the $L^{p}$-duality underlying the representation of monetary risk measures to a more flexible Orlicz duality, in spaces of stochastic processes modelling random future evolution of financial values in continuous time over a finite horizon. This contributes, on the one hand, to the theory of real-valued monetary risk measures for processes and, on the other hand, supports a new representation of acceptability indices of financial performance.
\end{abstract}

Keywords Concave monetary utility functionals · Monetary risk measures for processes · Orlicz space duality · Acceptability indices

JEL Classification C02 · C44 · G11 · G20

\section{Introduction}

Coherent measures of risk for processes as introduced in Cheridito et al. (2004) generalize the well-developed point-in-time framework for quantifying market risk. The main idea is to define a mapping acting on suitable spaces of stochastic processes modelling possible realizations of cash flow over a finite time horizon. Practically, the need of such risk measure may be attributed to regulatory institutions who want to compute the numerical value of losses potentially incurred at any time of a trading horizon. Indeed, since the early 1990s, the point-in-time value at risk, defined as quantile of a profit and loss probability distribution at the end of a fixed trading horizon,

\footnotetext{
Damiano Rossello

rossello@unict.it

Christos E. Kountzakis

chr_koun@aegean.gr

1 Department of Mathematics, University of the Aegean, 83200 Samos, Greece

2 Department of Economics and Business, University of Catania, 95129 Catania, Italy
} 
has been a widely used quantitative measure of market risk for regulatory purposes (cf. Basel Committee on Banking Supervision 2006 and Basel Committee on Banking Supervision 2019). But major deficiencies of this risk measure are well known: firstly, the actual size of losses is ignored; secondly it is not subadditive, then violating the axiomatic definition of coherence in the sense of Artzner et al. (1999); lastly, it does not account for the whole magnitude of potential losses within a trading horizon. The first two items have been addressed by introducing the expected shortfall risk measure, which is coherent and sensitive to the tail of the profit and loss distribution (see Artzner et al. 1999; Acerbi and Tasche 2002; Rockafeller and Uryasev 2002). Moreover, the recent market risk framework of the Basel Accords admits the expected shortfall as an adequate risk measure because of its ability in providing sufficiently conservative risk estimates. This and a constant demand for quantitative measures which depends on all extremes in a trading horizon push forward the recent academic contributions on the subject. In particular, considering the running minimum of a profit and loss process and applying the point-in-time definition of value at risk to the resulting realvalued distribution have been recently studied in Bhattacharyya et al. (2009), Bakshi and Panayotov (2010), Boudoukh et al. (2004) and termed as intra-horizon risk. Other directions have been explored for example by Goldberg and Mahmoud (2017) where intra-horizon risk measures are in connection with the well-known maximum drawdown. However, all these risk measures are not coherent in the sense of Cheridito et al. (2004). A possible remedy to the lack of coherence has been recently studied in Farkas et al. (2020), replacing the point-in-time expected shortfall to the distribution of the profit and loss's running minimum.

Along the lines of the above theoretical and practical issues, the current article describes the duality $\left\langle\mathscr{R}^{\infty}, \mathscr{A}^{1}\right\rangle$, and in general the transfer of the $L^{p}$-duality to spaces of stochastic processes through the dual pairs $\left\langle\mathscr{R}^{p}, \mathscr{A}^{q}\right\rangle$, for conjugate exponents $p, q \in[0,+\infty]$. The set $\mathscr{R}^{p}$ for $p \in[1,+\infty]$ contains càdlàg processes satisfying a moment condition concerning the running maximum of the reflected process, see Sect. 2 . In particular, the set $\mathscr{R}^{\infty}$ contains bounded càdlàg processes, while $a \in \mathscr{A}^{1}$ are bi-variate stochastic processes acting as integrators in the bi-linear form $\langle X, a\rangle$, see Cheridito et al. (2004) and Sect. 2. The proper choice of $a \in \mathscr{A}^{1}$ gives raise to interesting examples of risk measurement for processes, see (Cheridito et al. 2004, Section 5). The space $\mathscr{A}^{q}$ generalizes to the case $q>1$ for conjugate exponents $p^{-1}+q^{-1}=1$, and contains bi-variate processes satisfying a moment condition concerning the variation of both components, see again Sect. 2. In view of all these premises, the purpose of our paper is twofold:

- Given two Orlicz spaces of stochastic processes $\mathscr{R}^{\Phi}, \mathscr{A}^{\Psi}$, with corresponding conjugate Young functions $\Phi, \Psi$, we define Orlicz duality by the dual pair $\left\langle\mathscr{R}^{\Phi}, \mathscr{A}^{\Psi}\right\rangle$;

- We define convex and coherent monetary risk measures on these spaces and then provide their representation accordingly. For the coherent case, we additionally provide a representation result for special classes of financial performance measures.

The reason for using Orlicz spaces in finance, and more specifically in risk analysis, is highlighted if we recall the set-inclusion scheme as provided, for example, by 
(Cheridito and Li 2009, Example 4.1.3):

$$
L^{\infty} \subset M^{\Phi} \subset L^{p} \subset L^{\Psi} \subset L^{1}
$$

for any $p \in(1, \infty)$ and every $\lambda>0$, where $\Phi(x)=\mathrm{e}^{\lambda x}-1$ induces the Orlicz norm $\|\cdot\|_{\Phi}$ and similarly

$$
\Psi(y)= \begin{cases}0, & \text { if } y \leqslant \lambda \\ \frac{y}{\lambda} \log \left(\frac{y}{\lambda}\right)-\frac{y}{\lambda}+1, & \text { if } y>\lambda\end{cases}
$$

induces the Orlicz norm $\|\cdot\|_{\Psi}$. The families $L^{\Phi}$ and $L^{\Psi}$ contain those random variables describing discounted future net worth for which $\|\cdot\|_{\Phi}$ and $\|\cdot\|_{\Psi}$ are finite. Recall that $L^{\Psi}$ is an Orlicz space and $M^{\Phi}$ is the Orlicz heart ${ }^{1}$ of the conjugate Orlicz space $L^{\Phi}$. Since any Orlicz norm might be used to estimate the tail of a probability distribution, especially if based on exponential functions which give much more weight in the tails, the above set-inclusions imply that every random variable in $L^{1}$ having finite Kullback-Leibler divergence (hence finite relative entropy) under different probability measures is a non-heavy tailed random variable. This also implies that the use of Orlicz duality far from $L^{p}$ spaces has a specific risk-analytic meaning: The Young function $\Phi$ of the Orlicz heart $M^{\Phi}$ represents a 'moment-function', while the conjugate Young function $\Psi$ of the Orlicz space $L^{\Psi}$ can be interpreted as a risk functional. See "Appendix A" for a quick review of Orlicz spaces. In words, since models in finance and insurance as profits/losses at a terminal date or cumulative returns are better described by unbounded random variables and they can be derived by corresponding unbounded stochastic processes, our contribution is to define risk measures for processes whose paths are not necessarily bounded. To this scope, we use the dual pair $\left\langle\mathscr{R}^{\Phi}, \mathscr{A}^{\Psi}\right\rangle$ where in general the moment condition satisfied by processes belonging to $\mathscr{R}^{\Phi}$ is based on the Luxemburg norm $\|\cdot\|_{\Phi}$ and $\mathscr{R}^{\Phi}$ acts as an Orlicz heart modelling unbounded financial paths such that $\mathscr{R}^{\infty} \subseteq \mathscr{R}^{\Phi} \subset \mathscr{R}^{0}$. We do not pursue the extension argument of Cheridito et al. (2006) from $\mathscr{R}^{\infty}$ to $\mathscr{R}^{0}$. For the sake of completeness, notice that $\mathscr{A}^{q} \subseteq \mathscr{A}^{\Psi} \subseteq \mathscr{A}^{1}$ for conjugate exponents $p, q$ (for the exact definition of $\mathscr{A}^{\Psi}$ see Sect. 2).

The present article is organized as follows: Section 2 sets up the proper spaces of stochastic processes to work with. In particular, Sect. 2.1 adequately motivates our contribution with respect to that of Cheridito and Li (2009). In Sect. 3, risk measures on Orlicz spaces of stochastic processes are defined, and robust representation results are provided. Section 4 yields the connection between such risk measures and acceptability indices of performances for processes. The financial and mathematical duality is formally established, and a representation result is proved. Moreover, the differences between the current contribution and that of Cherny and Madan (2009) are pointed out. Section 5 contains some concluding remarks.

\footnotetext{
${ }^{1}$ Actually, a maximal subspace of Orlicz classes which includes $L^{p}$ spaces for $p \in[1, \infty)$.
} 


\section{Notations and Definitions}

We model the evolution of a (discounted) cash flow over a finite time-interval rather than handling only terminal cash flows. A stochastic process $X=\left(X_{t}\right)_{t \in[0, T]}$ serves to the scope, for each scenario resulting from trading over the investment horizon $[0, T]$, where $T \in(0, \infty)$. Its realizations can be interpreted as possible (discounted) profit and loss realizations of a given financial position over the valuation time interval. Except the setting of Sect. $4, X$ does not represent necessarily a whole zero-cost selffinanced portfolio, rather it can be understood as a profit and loss process starting at $X_{0}=z$ where $z \in \mathbb{R}$ represents the profit/loss accumulated until the start of the time horizon, since profits/losses from a financial position can be rolled over multiple valuation periods.

The stochastic base $(\Omega, \mathscr{F}, \mathbf{F}, \mathrm{P})$, where $\mathbf{F}=\left(\mathscr{F}_{t}\right)_{t \in[0, T]}$, satisfies the usual conditions, namely the probability space $(\Omega, \mathscr{F}, \mathrm{P})$ is complete, the filtration $\mathbf{F}$ is right-continuous, and the sub- $\sigma$-algebra $\mathscr{F}_{0}$ contains all the P-null sets of $\mathscr{F}$. As usual, we set $L^{p}:=L^{p}(\Omega, \mathscr{F}, \mathrm{P})$ for $p \in[0,+\infty]$. The vector space of càdlàg and F-adapted processes is denoted by $\mathscr{R}^{0}$. Almost surely (a.s.) equal random variables are identified as well as indistinguishable processes on the filtered space, $X_{t}(\omega)=Y_{t}(\omega)$ is understood P-a.s. and for all $t \in[0,+\infty]$. The usual partial ordering on $\mathscr{R}^{0}$ is defined as:

$$
X \geqslant Y \Longleftrightarrow X_{t}(\omega) \geqslant Y_{t}(\omega), \quad \text { for every } t \in[0,+\infty] \text { and all } X, Y \in \mathscr{R}^{0} \text {. }
$$

Let $X^{*}:=\sup _{t \in[0, T]}\left|X_{t}\right|$. If $\Phi$ is a Young function, we may define the following Banach lattice of stochastic processes:

$$
\mathscr{R}^{\Phi}:=\left\{X \in \mathscr{R}^{0} \mid\left\|X^{*}\right\|_{\Phi}<\infty\right\}
$$

where $\|\cdot\|_{\Phi}$ is the Luxemburg norm acting on the associated Orlicz space $L^{\Phi}$. For the conjugate Young function $\Psi$, we introduce the following space of bi-variate stochastic processes:

$$
\mathscr{A}^{\Psi}:=\left\{\begin{array}{l|l}
a:[0, T] \times \Omega \rightarrow \mathbb{R}^{2} & \begin{array}{l}
a=\left(a_{1}, a_{2}\right) \text { right-continuous, finite variation } \\
a_{1} \text { predictable, } a_{1,0}=0 \\
a_{2} \text { optional, purely discontinuous } \\
\operatorname{Var}\left(a_{1}\right)+\operatorname{Var}\left(a_{2}\right) \in\left(L^{\Phi}\right)^{*}
\end{array}
\end{array}\right\}
$$

where $\left(L^{\Phi}\right)^{*}$ is the dual space of $L^{\Phi}$, which is either $L^{\Psi}$ or $M^{\Psi}$. Recall that $X \in \mathscr{R}^{0}$ is optional whenever it is measurable on $[0, T] \times \Omega$ equipped with the $\sigma$-algebra generated by the all the members of $\mathscr{R}^{0}$; if $X$ is measurable on $[0, T] \times \Omega$ equipped with the $\sigma$-algebra generated by the adapted left-continuous processes, then it is predictable. Hence, an increasing process $a_{i}:[0, T] \times \Omega \rightarrow \mathbb{R}$ (i.e. $\mathbf{F}$-adapted, with positive right-continuous and increasing paths) induces a measure $\mathrm{d} a_{i, t}(\omega)$. In case $a_{i}$ has right-continuous paths with finite variation, its unique decomposition $a_{i}=a_{i}^{+}-a_{i}^{-}$ into two right-continuous increasing processes induces two $\mathrm{P}$-a.s. positive measures on 
$[0, T]$ with disjoint support. The total variation of such process is the random variable $\operatorname{Var}\left(a_{i}\right):=a_{i, T}^{+}+a_{i, T}^{-}$. Moreover, if $a_{i}$ is optional, then $a_{i}^{+}, a_{i}^{-}$are too. When $a_{i}$ is predictable, then $a_{i}^{+}, a_{i}^{-}$are too. The duality between $\mathscr{R}^{\Phi}$ and $\mathscr{A}^{\Psi}$ is established through the bi-linear form

$$
\langle X, a\rangle:=\mathrm{E}\left[\int_{(0, T]} X_{t^{-}} \mathrm{d} a_{1, t}+\int_{[0, T]} X_{t} \mathrm{~d} a_{2, t}\right],
$$

such that $|\langle X, a\rangle| \leqslant\|X\|_{\mathscr{R}^{\Phi}}\|a\|_{\mathscr{A}^{\Psi}}$, where $^{2}$

$$
\|X\|_{\mathscr{R}^{\Phi}}:=\left\|X^{*}\right\|_{\Phi}
$$

and

$$
\|a\|_{\mathscr{A}^{\Psi}}:=\left\|\operatorname{Var}\left(a_{1}\right)+\operatorname{Var}\left(a_{2}\right)\right\|_{\Psi} .
$$

\subsection{Motivation of the Paper}

Our contribution in the present article is different from that of Cheridito and $\mathrm{Li}$ (2009) for two reasons. First, the representation of $(-\infty,+\infty]$-valued convex and coherent monetary risk measures for possibly unbounded càdlàg processes $X \in \mathscr{R}^{\Phi}$ we provide in Sect. 3.1 can be reduced to the case of random variables on Orlicz hearts provided that $T=0$. Indeed, this entails $\mathscr{R}^{\Phi}=M^{\Phi}$ and $\mathscr{A}^{\Psi}=L^{\Psi}$. The Orlicz spaces of stochastic processes $\mathscr{R}^{\Phi}, \mathscr{A}^{\Psi}$ are Banach lattices, but in our proofs of the main representation results (see Theorems 1 and 2) we do not use neither the monotonicity of functionals on Banach lattices whose domain has non-empty interior equal to their algebraic interior, nor any growth condition satisfied by the penalty function used in the robust representation (for the definition of a penalty function in the current context see Sect. 3.1). Observe that in general, $M^{\Phi} \subseteq L^{\Psi}$ but $\mathscr{R}^{\Phi} \nsubseteq \mathscr{A}^{\Psi}$ even if $\mathscr{R}^{p}$ can be identified with the topological dual of $\mathscr{A}^{q}$, for conjugate exponents $p, q$. Moreover, by (Cheridito et al. 2004, Remark 3.9) the Orlicz heart $\mathscr{R}^{\Phi} \subseteq \mathscr{R}^{p}$ is different for each probability measure governing the underlying random mechanism, which can be interpreted as a subjective modelling of risk measures for processes. Second, for $T>0$ only in our setting it is possible to start from a stochastic process whose paths model the evolution of profits and losses or cumulative returns over a finite trading horizon. To be more specific, let $\tilde{\rho}: M^{\Phi} \rightarrow \mathbb{R}$ be a convex monetary risk functional acting on random variables $\underline{X} \in M^{\Phi}$ modelling possibly unbounded cash flows, represented as

$$
\tilde{\rho}(\underline{X})=\sup _{Z \in A}\left\{E_{p}(\underline{X} \cdot Z)-\tilde{\gamma}(Z)\right\}
$$

$\overline{{ }^{2} \text { For } p=\infty \text { and } q=1 \text { one has }}\|X\|_{\mathscr{R}} \infty:=\left\|X^{*}\right\|_{\infty}$ and $\|a\|_{\mathscr{A} 1}:=\left\|\operatorname{Var}\left(a_{1}\right)+\operatorname{Var}\left(a_{2}\right)\right\|_{1}$. 
where $A$ is the set containing those nonnegative random variables with $\mathrm{E}_{\mathrm{p}}(Z)=1$, for some penalty term $\tilde{\gamma}: A \rightarrow[-\infty,+\infty)$. Thus, define

$$
\gamma(a):= \begin{cases}\tilde{\gamma}(Z), & \text { if } a=\left(0, Z \mathbf{1}_{\{\theta \leqslant t\}}\right) \text { for some } Z \in A \\ & \text { and a }[0, T]-\text { valued random variable } \theta \\ -\infty, & \text { otherwise. }\end{cases}
$$

Then, by (Cheridito et al. 2004, Remark 3.7) together with (Cheridito et al. 2004, Proposition 8, Remark 3.9) and Theorem 1, we have

$$
\rho(X)=\inf _{a \in \mathscr{B}_{1}}\{\langle X, a\rangle-\gamma(a)\}=\tilde{\rho}\left(\inf _{t \in[0, T]} X_{t}\right),
$$

for stochastic processes $X \in \mathscr{R}^{\Phi}$. As a consequence, the approach of Cheridito and Li (2009) cannot handle directly a whole path generating by a trading strategy over a finite time horizon. It is worth noting that the set $\mathscr{B}_{1}$ can be identified with $\mathscr{D}_{\sigma} \cap \mathscr{A}^{\Psi}$, where the set $\mathscr{D}_{\sigma}$ used in Cheridito et al. (2004) contains those bi-variate processes $a \in \mathscr{A}_{+}^{1}$ such that $\|a\|_{\mathscr{A}^{1}}=1$.

The practical relevance of our approach is to deal with processes representing possible paths of profits and losses from a position or portfolio, as derived by Brownian motion, jump-diffusion processes of more general Lévy processes, and accounting for intrahorizon risk rather than merely expected end-of-horizon loss. In fact, for the noncoherent case, Bakshi and Panayotov (2010) already pointed out the importance of quantify the magnitude of losses within a trading horizon, especially in a mark-tomarket environment. But differently from Bakshi and Panayotov (2010) we can handle intra-horizon risk using convex and in particular coherent risk measure for processes. For example, choosing the Young function $\Phi(x)=x^{p}$ for $p \in(1,+\infty)$, we have $\mathscr{R}^{\Phi}=\mathscr{R}^{p}$ and $\mathscr{A}^{\Psi}=\mathscr{A}^{q}$, for conjugate exponents $p, q$ so that by the reasoning above we get the coherent risk measure for processes

$$
\rho(X):=-\frac{1}{\beta} \int_{0}^{\beta} \operatorname{VaR}_{c}\left(\inf _{t \in[0, T]} X_{t}\right) \mathrm{d} c
$$

for any $\beta \in(0,1)$, where $\operatorname{VaR}_{c}(\cdot)$ is the usual point-in-time value at risk and $\inf _{t \in[0, T]} X_{t}$ is the running minimum of the underlying position over the horizon. In this case, we have that $\tilde{\rho}$ above is the usual expected shortfall risk measure, which is applied to a path-transformation given by the running minimum. In contrast to our approach, Boudoukh et al. (2004), Bakshi and Panayotov (2010) and Bhattacharyya et al. (2009) use the non-coherent risk measure for processes based only on the static value at risk, what the authors called intra-horizon risk measure. For a more recent contribution based on the point-in-time expected shortfall as a risk functional, combined with the path-transformation given by the running minimum, see Farkas et al. (2020), where in addition an interesting connection with first-passage probabilities is established. 


\section{Risk measures on Orlicz spaces of processes}

In this section, we apply the special Orlicz spaces previously introduced to obtain a new definition of risk measures for processes. Our approach as in Cheridito et al. (2004) is to look at a whole path modelling net worths or financial returns over a fixed horizon and then deduce a risk quantification which is by no mean truly dynamic but nevertheless is able to account for the entire history of profits and losses of a traded position. Since we use a static risk measure acting on a proper domain of financial path (i.e. stochastic processes picked from the corresponding Orlicz space), it is beyond the scope of this paper to treat properties possessed by dynamic risk measures such as time-consistency.

Definition 1 (General Properties) A monetary risk measure for processes is a mapping $\rho: \mathscr{R}^{\Phi} \rightarrow \mathbb{R}$ satisfying the following two properties:

(M) Monotonicity $\rho(X) \leqslant \rho(Y)$ for all $X, Y \in \mathscr{R}^{\Phi}$ such that $X \geqslant Y$

(T) Translation invariance $\rho(X+m \mathbf{1})=\rho(X)-m$ for all $X \in \mathscr{R}^{\Phi}$ and $m \in \mathbb{R}$, where $\mathbf{1}=\left(\mathbf{I}_{\{t\}}\right)_{t \in[0, T]}$ is the process equal to 1 at any date within the horizon, i.e. $\mathbf{I}_{\{t\}}=1$ at time $t$ and zero elsewhere.

We call a monetary risk measure for processes convex if it also satisfies

(C) Convexity $\rho(\lambda X+(1-\lambda) Y) \leqslant \lambda \rho(X)+(1-\lambda) \rho(Y)$ for all $X, Y \in \mathscr{R}^{\Phi}$ and $\lambda \in(0,1)$.

A convex monetary risk measure for processes is called coherent if it fulfils

(P) Positive homogeneity $\rho(\lambda X)=\lambda \rho(X)$ for all $X \in \mathscr{R}^{\Phi}$ and $\lambda \in \mathbb{R}_{+}$.

Observe that under $(\mathrm{P})$, property $(\mathrm{C})$ is equivalent to

(S) Subadditivity $\rho(X+Y) \leqslant \rho(X)+\rho(Y)$, for all $X, Y \in \mathscr{R}^{\Phi}$.

We also refer to concave monetary utility function $f: \mathscr{R}^{\Phi} \rightarrow \mathbb{R}$ which is the negative of a convex monetary risk measure for processes, $f=-\rho$. Whenever $\rho$ is coherent, we call $f$ coherent too.

Remark 1 The stochastic process $\mathbf{1}$ is a strictly positive functional on the cone $\mathscr{A}_{+}^{\Psi}$, which contains the bi-variate increasing processes of $\mathscr{A}^{\Psi}$ with nonnegative optional and purely discontinuous components, $a_{1}, a_{2} \geqslant 0$. By $\mathscr{B}_{1}$ we denote the base defined by $\mathbf{1}$ on the cone: $\mathscr{B}_{1}=\left\{a \in \mathscr{A}_{+}^{\Psi} \mid\langle\mathbf{1}, a\rangle=1\right\}$. This behaves as the set of RadonNykodym derivatives in the robust representation of a point-in-time monetary risk measure. Observe that the set $\mathscr{A}_{+}^{\Psi}$ can be identified with the topological dual $\left(\mathscr{R}^{\Phi}\right)^{*}$ of the Orlicz heart $\mathscr{R}^{\Phi}$.

\subsection{Representation results}

We are in position to establish a robust representation for convex and coherent risk measures for processes, using the Orlicz duality introduced so far. We also obtain strong and weak continuity of such convex and coherent risk measures for processes, 
under some additional assumptions. A penalty function $\gamma: \mathscr{B}_{1} \rightarrow \overline{\mathbb{R}}$ corresponding to a convex risk measure $\rho$, which admits a $\sigma\left(\mathscr{R}^{\Phi}, \mathscr{A}^{\Psi}\right)$-closed acceptance set $\mathcal{C}_{\rho}=$ $\left\{X \in \mathscr{R}^{\Phi} \mid \rho(X) \leqslant 0\right\}$, is defined for a fixed bi-variate process $a \in \mathscr{B}_{1}$ as

$$
\gamma(a):=\sup \left\{\langle-X, a\rangle-\rho(X) \mid X \in \mathscr{R}^{\Phi}\right\} .
$$

Here, $\gamma(a)$ is the conjugate function of $\rho(X)$. It is easily shown that $\gamma(a) \in$ $(-\infty,+\infty]$ for all $a \in \mathscr{B} 1$. Thanks to the characterization of the penalty function and to Lemma 1 (see "Appendix C"), we may provide the following two representation results for convex and coherent risk measures, respectively.

Theorem 1 If $\rho: \mathscr{R}^{\Phi} \rightarrow \mathbb{R}$ is a convex risk measure for processes, then

$$
\rho(X)=\sup \left\{\langle-X, a\rangle-\gamma(a) \mid a \in \mathscr{B}_{1}\right\}
$$

for every $X \in \mathscr{R}^{\Phi}$.

Before stating the following theorem and subsequent results, we recall that for a coherent $\rho$ the cone $\mathcal{C}_{\rho}$ has a polar set $\mathcal{C}_{\rho}^{0}:=\left\{a \in \mathscr{A}^{\Psi} \mid\langle X, a\rangle \geqslant 0\right\}$, for every acceptable $X \in \mathcal{C}_{\rho}$.

Theorem 2 If $\rho: \mathscr{R}^{\Phi} \rightarrow \mathbb{R}$ is a coherent risk measure for processes and $\mathcal{C}_{\rho}$ is $\sigma\left(\mathscr{R}^{\Phi}, \mathscr{A}^{\Psi}\right)$-closed, then

$$
\rho(X)=\sup \left\{\langle-X, a\rangle \mid a \in \mathscr{B}_{1} \cap \mathcal{C}_{\rho}^{0}\right\}
$$

for every $X \in \mathscr{R}^{\Phi}$.

The connection between convex monetary risk measures for processes and concave monetary utility functions is given below, see Delbaen (2012).

Corollary 1 A concave monetary utility function $f: \mathscr{R}^{\Phi} \rightarrow \mathbb{R}$ with $\sigma\left(\mathscr{R}^{\Phi}, \mathscr{A}^{\Psi}\right)$ closed upper-level set $\mathcal{C}_{f}=\left\{X \in \mathscr{R}^{\Phi} \mid f(X) \geqslant 0\right\}$, admits the dual representation

$$
f(X)=\inf \left\{\langle X, a\rangle+\gamma(a) \mid a \in \mathscr{B}_{1}\right\}
$$

where $\gamma(a)=-\inf \left\{\langle X, a\rangle \mid X \in \mathcal{C}_{f}\right\}$.

Eventually, we provide the following couple of continuity results.

Theorem 3 A convex risk measure for processes $\rho: \mathscr{R}^{\Phi} \rightarrow \mathbb{R}$ as given in Theorem 1 is a Lipschitz function, provided that 1 defines a bounded base on $\mathscr{A}_{+}^{\Psi}$. Then, $\rho$ is norm and weakly continuous.

Theorem 4 A coherent risk measure for processes $\rho: \mathscr{R}^{\Phi} \rightarrow \mathbb{R}$ as given in Theorem 2 is a Lipschitz function, provided that $\mathbf{1}$ defines a bounded base on $\mathscr{A}_{+}^{\Psi}$. Then, $\rho$ is norm and weakly continuous. 


\section{Monetary risk measures for processes and acceptability indices}

The balance between reward and risk associated with a given investment strategy is a central theme in the industry of managed funds. Performance measures have been tailored to quantify this balance. Cherny and Madan (2009) provided a first unified framework to define the mathematical properties of such measures, beyond the classical Sharpe ratio expressed as a reward-to-variability index of performance (i.e. expected excess return divided by standard deviation). In fact, risk-adjusted return on capital (RAROC) is built upon the ratio of mean excess return to some different measure of risk. Based on a point-in-time perspective, Cherny and Madan (2009) treat a performance measure as an acceptability index (AI) based on some desirable properties emphasizing the "duality" between AIs and coherent risk measures for random variables modelling terminal cash flows.

In this section, the process $X \in \mathscr{R}^{\Phi}$ models the realizations of a (discounted) cash flow over a valuation horizon $[0, T]$, possibly as profits and losses even in percentage form; $X$ is produced by a zero-cost self-financed portfolio. There is a transition from a pointin-time performance measure for a random variable representing terminal cash flows, to the process $X$. We claim that a static index of performance $\alpha$ acts on processes and yields numerical values, requiring a coherent risk measure which records all possible stressed scenarios during the horizon. A larger $\alpha$ means better performance: the larger the reward associated with the $X$ 's paths (adjusted for a coherent risk measure for processes at any time within the horizon) the higher its degree of acceptability. A performance $\alpha$ valued at $+\infty$ can be understood as an "arbitrage opportunity", in particular if $X_{t}(\omega)>0$ for every $t \in[0, T]$ and $\omega \in \Omega$, see the concept of arbitrage consistency stated in Sect. 4.1. By means of the $X$ 's realizations, we are able as well to retain all the information involved as time elapses. In this section, we use Theorems 2 and 4 to establish a duality relationship between coherent risk measures for stochastic processes defined over Orlicz spaces, and performance measures given over the same spaces. Our representation of an AI is not meant to be dynamic, since it relies on coherent monetary risk measures yielding a numerical evaluation rather than a sequence of conditional risk measures (random variables). Indeed, dynamic risk measures account for the information available at the risk assessment when (on some filtered probability space) it is updated according to different notions of time consistency, see Acciaio and Penner (2011) and the references therein.

Before to state the main theorem, we formally set the axiomatic definition of an AI for processes.

Definition 2 A map $\alpha: \mathscr{R}^{\Phi} \rightarrow[0,+\infty]$ is an acceptability index for processes if it satisfies the following three properties. Quasi-concavity given a pair $X, Y \in \mathscr{R}^{\Phi}$ and for every $\lambda \in[0,1]$ such that $\alpha(X) \geqslant x$ and $\alpha(Y) \geqslant x$ it holds

$$
\alpha(\lambda X+(1-\lambda) Y) \geqslant x
$$

Monotonicity for any $X, Y \in \mathscr{R}^{\Phi}$

$$
X \leqslant Y \Rightarrow \alpha(X) \leqslant \alpha(Y)
$$


Scale invariance for every $\lambda>0$ and $X \in \mathscr{R}^{\Phi}$

$$
\alpha(\lambda X)=\alpha(X)
$$

Quasi-concavity is equivalent to the convexity of the acceptance set

$$
\mathscr{C}_{x}^{\alpha}:=\left\{X \in \mathscr{R}^{\Phi} \mid \alpha(X) \geqslant x\right\}
$$

Thus, a diversified position performs better than its components since $x=$ $\min \{\alpha(X), \alpha(Y)\}$. Moreover, acceptable cash flows are valued monotonically: provided $X$ is dominated by $Y$ then $\alpha$ is an increasing and $Y$ is at least as acceptable as $X$. By scale invariance any acceptance set $\mathscr{C}_{x}^{\alpha}$ is a convex cone: $\alpha$ measures to what extent moving away from marginal trades underlying the random cash flow $X$ results in a new investment direction based on alternative pricing equations. The performance of an investment should not depend upon the initial endowment because $\lambda X$ is based on a trade in the same direction of $X$ and has the same level of acceptance. Notice that, compared with (Cherny and Madan 2009, Axiom 1.4), we do not require the Fatou property. For the case of bounded realizations of $X$, see Kountzakis and Rossello (2020). The relationship between risk measures and AIs in the current setting is better understood if we recall the analogous duality between static AIs defined for $X \in L^{\infty}$ as

$$
\alpha(X)=\sup \left\{x \in \mathbb{R}_{+} \mid \inf _{\mathbb{Q} \in \mathscr{D}_{x}} \mathrm{E}_{Q}(X) \geqslant 0\right\}
$$

see (Cherny and Madan 2009, Theorem 1), and static coherent risk measures $\rho(X)=$ $\sup \left\{\mathrm{E}_{Q}(-X) \mid \mathbb{Q} \in \mathscr{D}\right\}$, where $\left(\mathscr{D}_{x}\right)_{x \in \mathbb{R}_{+}}$is a family of sets of probability measures (increasing in $x$ ) such that $\mathscr{D} x \subset \mathscr{D}$ and $\mathscr{D}$ is a certain set of probability measures absolutely continuous with respect to $P$. Indeed, it is easily seen

$$
\alpha(X)=\sup \left\{x \in \mathbb{R}_{+} \mid \rho_{x}(X) \leqslant 0\right\}, \text { for every } X \in L^{\infty} .
$$

Now, coherent risk measures represented as in Theorem 2 of the current article can be indexed by $x \in \mathbb{R}_{+}$. Hence, $x \mapsto \rho_{x}(X)$ is a map evaluating the performance of a trading strategy over the entire horizon and for every acceptability level $x>0$. For a family $\left(\rho_{x}(X)\right)_{x \in \mathbb{R}_{+}}$of such coherent risk measures any member is required to be increasing in $x$,

$$
\rho_{x}(X) \leqslant \rho_{y}(X), \quad \text { for every } 0<x \leqslant y, \quad X \in \mathscr{R}^{\Phi} \quad \text { fixed. }
$$

It suffices to let

$$
\rho_{x}(X):=\inf \{m \in \mathbb{R} \mid \alpha(X+m \mathbf{1}) \geqslant x\}, \quad \text { for every } x \in \mathbb{R}_{+}, X \in \mathscr{R}^{\Phi},
$$

and take the infimum over $m \in \mathbb{R}$ of both sets

$$
\{m \in \mathbb{R} \mid \alpha(X+m \mathbf{1}) \geqslant y\} \subset\{m \in \mathbb{R} \mid \alpha(X+m \mathbf{1}) \geqslant x\},
$$


for any $0<x \leqslant y$. Any map in this increasing family can be represented via an AI for processes, see Lemma 2 in "Appendix C". Moreover, we can represent AIs in terms of an increasing family of coherent risk measures for processes, see Lemma 3 in "Appendix C". Hence, we have the following duality relationship between coherent risk measures and AIs for processes:

Corollary 2 (a) If $\alpha: \mathscr{R}^{\Phi} \rightarrow[0,+\infty]$ is an AI for processes, then there exists an increasing family $\left(\rho_{x}\right)_{x \in \mathbb{R}_{+}}$of coherent risk measures for processes such that the following representation holds:

$$
\alpha(X):=\sup \left\{x \in \mathbb{R}_{+} \mid \rho_{x}(X) \leqslant 0\right\}
$$

(b) If $\left(\rho_{x}\right)_{x \in \mathbb{R}_{+}}$is an increasing family of coherent risk measures for processes, then there exists an AI for processes such that for every $x \in \mathbb{R}_{+}$the following representation holds:

$$
\rho_{x}(X):=\inf \{m \in \mathbb{R} \mid \alpha(X+m \mathbf{1}) \geqslant x\}, \text { for every } X \in \mathscr{R}^{\Phi},
$$

where inf $\varnothing=+\infty$ and $\sup \varnothing=0$.

A subset of a vector space is a wedge if it is convex and it has the property that for any element lying in the set we also have that its scaled version (by a nonnegative constant) belongs to the same set. Second, an indexed family of sets $\left(G_{x}\right)_{x \in \mathbb{R}_{+}}$is increasing if $x \leqslant y$ implies $G_{x} \subset G_{y}$, for every $x, y \in \mathbb{R}_{+}$.

Theorem 5 Let $\alpha: \mathscr{R}^{\Phi} \rightarrow[0,+\infty]$ be an AI with acceptability sets $\mathscr{C}_{x}^{\alpha}=\{X \in$ $\left.\mathscr{R}^{\Phi} \mid \alpha(X) \geqslant x\right\}$, for every $x>0$, being wedges. Then, there exists an increasing family $\left(G_{x}\right)_{x \in \mathbb{R}_{+}} \subset \mathscr{A}^{\Psi}$ such that

$$
\alpha(X)=\sup \left\{x \in \mathbb{R}_{+} \mid \inf _{g \in G_{x}}\langle X, g\rangle \geqslant 0\right\},
$$

with inf $\varnothing=+\infty$ and $\sup \varnothing=0$.

To clarify the practical relevance of the above result, we provide the following example. Since each set $G_{x}$ in the representation of AIs as in Theorem 5 can be identified with a subset of $\mathscr{D}_{\sigma} \cap \mathscr{A}^{\Psi}$ (see ${ }^{3}$ Sect. 2.1), we pick the bi-variate process

$$
\tilde{a}:=\frac{1}{1+x} b+\frac{x}{1+x} a, \quad x \in \mathbb{R}_{+}, \quad a, b \in \mathscr{D}_{\sigma} \cap \mathscr{A}^{\Psi}
$$

where

$$
b=\left(b_{1, t}, b_{2, t}\right)_{t \in[0, T]}:=\left(0, \mathbf{1}_{\{u \leqslant t\}}\right)_{t \in[0, T]} .
$$

\footnotetext{
$\overline{3}$ Essentially, each $G_{x}$ can be viewed as a subset $\mathscr{B}_{x} \subset \mathscr{B}$, such that $\left(\mathscr{B}_{x}\right)_{x \in \mathbb{R}_{+}}$is again an $x$-increasing family.
} 
We have the following chain of equivalences:

$$
\begin{aligned}
\alpha(X) \geqslant x & \Longleftrightarrow \frac{\mathrm{E}\left(X_{T}\right)}{\rho(X)} \geqslant x \\
& \Longleftrightarrow \mathrm{E}\left(X_{T}\right) \geqslant-x \cdot \inf _{a \in \mathscr{D}_{\sigma} \cap \mathscr{A}^{\Psi}}\langle X, a\rangle \\
& \Longleftrightarrow \frac{1}{1+x} \mathrm{E}\left(X_{T}\right)+\frac{x}{1+x} \inf _{a \in \mathscr{D}_{\sigma} \cap \mathscr{A}^{\Psi}}\langle X, a\rangle \geqslant 0 \\
& \Longleftrightarrow \inf _{a \in \mathscr{D}_{\sigma} \cap \mathscr{A}^{\Psi}}\left[\frac{1}{1+x} \mathrm{E}\left(X_{T}\right)+\frac{x}{1+x}\langle X, a\rangle\right] \geqslant 0 \\
& \Longleftrightarrow \inf _{a \in \mathscr{D}_{\sigma} \cap \mathscr{A}^{\Psi}}\left\langle X, \frac{1}{1+x} \cdot b+\frac{x}{1+x} \cdot a\right\rangle \geqslant 0 \\
& \Longleftrightarrow \inf _{\tilde{a} \in G_{x}}\langle X, \tilde{a}\rangle \geqslant 0 .
\end{aligned}
$$

In the convex combination defining $\tilde{a}$, the first term $b$ projects the paths of profit/loss $X=\left(X_{t}\right)_{t \in[0, T]}$ onto the terminal date $T$ through the expectation; the second term $a$ pertains to the representation of the monetary risk measure for processes $\rho(X)$. Note that $\mathrm{E}\left(X_{T}\right)<+\infty$ by the assumption $X \in \mathscr{R}^{\Phi}$, for example by choosing $\Phi(x)=x^{p}$ with $p \in(1,+\infty)$. As by-product, the ratio

$$
\alpha(X)=\frac{\mathrm{E}\left(X_{T}\right)}{\rho(X)}
$$

is an AI for not necessarily bounded stochastic processes whose marginal distributions have finite $p$ th moments. Notice that by convention $\alpha(X)=+\infty$ whenever $\rho(X) \leqslant 0$, as in the static case. Therefore, our approach to AIs improves on the static one as given by Cherny and Madan (2009) because the latter handles one-dimensional random variables but is not suitable for random cash flows evolving over the horizon as represented by stochastic processes.

Remark 2 The above performance ratio $\alpha(X)$ is an AI whose risk measure can be specialized as a convex or a coherent risk measure for processes. For example, choosing the bi-variate process $a$ as we did in Sect. 2.1 one gets

$$
\rho(X)=\tilde{\rho}\left(\inf _{t \in[0, T]} X_{t}\right),
$$

and additionally considering the point-in-time weighted value at risk $\tilde{\rho}(\cdot)=$ WVAR(· ) used in (Cherny and Madan 2009, Section 3.6) this entails

$$
\tilde{\rho}(\underline{X})=-\int_{\mathbb{R}} y \mathrm{~d}\left(h_{x}\left(F_{\underline{X}}(y)\right)\right),
$$

where for every $x \in \mathbb{R}_{+}$the concave distortion $h_{x}(u):=\min \left\{\beta^{-1} u, 1\right\}$ is such that $\beta=1+x$, and for notational convenience $\underline{X}=\inf _{t \in[0, T]} X_{t}$ is the running 
minimum of the profit/loss $X$ with distribution function $F_{X}$. Observe that one also needs $\mathrm{E}_{\mathrm{P}}\left((Z-u)^{+}\right) \leqslant \tilde{h}_{x}(u)$ for every $u \in \mathbb{R}_{+}$such that $\tilde{h}$ is the convex conjugate of the concave distortion $h$, see (Cherny and Madan 2009, Section 3.6) for further details.

\subsection{Arbitrage consistency}

We provide a further result on arbitrage consistency. Intuitively, a position producing such a $X$ with a very high (positive) performance should be interpreted as an arbitrage opportunity, for every scenario and every time within the horizon. Given the duality between AIs and families of coherent risk measures for processes, as stated in Corollary 2 above, there is also a polar set $\mathscr{C}_{x}^{\alpha, 0} \subset \mathscr{R}^{\Phi}$ of the acceptability set $\mathscr{C}_{x}^{\alpha}$, for every $x \in \mathbb{R}_{+}$.

Proposition 1 Let $\alpha: \mathscr{R}^{\Phi} \rightarrow[0,+\infty]$ be an AI defined through a family of monotone, coherent risk measures for processes $\left(\rho_{x}\right)_{x \in \mathbb{R}_{+}}$in $\mathscr{R}_{+}^{\Phi}$ and the order unit 1 of $\mathscr{R}^{\Phi}$. Then, $\alpha$ is arbitrage consistent with respect to $\mathscr{R}_{+}^{\Phi}$.

For the sake of completeness, we link the above result to the theory of no arbitrage in continuous time. More specifically, we need the extension of the classical KrepsYan separation theorem to rule-out arbitrage opportunities, to Orlicz spaces of cash flow processes over the trading horizon. The subspace of those processes representing portfolio values which are replicable at time $T$ by a self-financing trading strategy with zero initial cost, is denoted by $\mathscr{K}$. The precise definition follows.

Theorem 6 Let $\Psi$ be some $N$-function and let $\left\langle\mathscr{A}^{\Psi}, \mathscr{R}^{\Phi}\right\rangle$ be the commodity-price duality, where $\mathscr{K}$ is the subspace of the replicated portfolio values satisfying the property of no-free lunch:

$$
{\overline{\left(\mathscr{K}-\mathscr{A}_{+}^{\Psi}\right)}}^{\sigma\left(\mathscr{A}^{\Psi}, \mathscr{R}^{\Phi}\right)} \cap \mathscr{A}_{+}^{\Psi}=\{0\}
$$

This implies the existence of some $f_{0} \in \mathscr{R}^{\Phi}$, such that $f_{0}(X)>0$, for every $X \in$ $\mathscr{A}_{+}^{\Psi} \backslash\{0\}$.

Theorem 6 is based, on the one hand, on standard arguments from the theory of Orlicz spaces, see Krasnoselski and Sobolev (1955) and Rao and Ren (1991), and, on the other hand, on arguments from the theory of no-arbitrage in continuous time. In particular, Theorem 6 is an Orlicz space's version of (Delbaen and Schachermayer 2006, Theorem 5.2.3) where the dual pair $\left\langle E, E^{*}\right\rangle$ is considered now in the special form $E=\mathscr{A}^{\Phi}$ and $E^{*}=\mathscr{R}^{\Phi}$. Recall that as a crucial ingredient in the proof, the Hahn-Banach theorem is in its version for separating hyperplanes. The following corollary arises from the fact that $\left(L^{\Phi}\right)^{*}=L^{\Phi}$, provided $\Phi$ both satisfies the $\Delta_{2}$-condition and is an $N$-function (see "Appendix A"), i.e. the topological dual $\left(\mathscr{A}^{\Phi}\right)^{*}$ of the space $\mathscr{A}^{\Phi}$ is just $\mathscr{R}^{\Phi}$.

Corollary 3 Let $\Phi$ be some $\Delta_{2}$-function and let $\left\langle\mathscr{A}^{\Phi}, \mathscr{R}^{\Phi}\right\rangle$ be the commodity-price duality, where $\mathscr{K}$ is the subspace of the replicated portfolio values, satisfying the 
property of no-free lunch:

$$
{\overline{\left(\mathscr{K}-\mathscr{A}_{+}^{\Phi}\right)}}^{\sigma\left(\mathscr{A}^{\Phi}, \mathscr{R}^{\Phi}\right)} \cap \mathscr{A}_{+}^{\Phi}=\{0\} .
$$

This implies the existence of some $f_{0} \in \mathscr{R}^{\Phi}$, such that $f_{0}(X)>0$, for every $X \in$ $\mathscr{A}_{+}^{\Phi} \backslash\{0\}$.

\section{Conclusions}

A recent research stream proposes to define monetary risk measures over spaces of appropriate stochastic processes instead of random variables, yielding a theoretical framework for special kinds of risk measures which are currently used in the finance industry to capture the magnitude of losses potentially incurred at any time of a trading horizon. We contribute to this theory by extending the seminal analysis in Cheridito et al. (2004) to the case of Orlicz spaces of processes modelling random cash flow that have not necessarily bounded realizations. Then, we apply coherent monetary risk measures for processes to acceptability indices of performance. Our approach depends upon mappings defined on Orlicz spaces of processes and paired via duality with proper conjugate spaces. Although these mappings entail numerical values and are not meant to be dynamic, we hope that our contribution could enrich the literature supporting the use of intra-horizon risk as recently treated in Farkas et al. (2020). We also try to provide an add-in to the corresponding literature on performance measures for processes as studied in Kountzakis and Rossello (2020).

Funding Open access funding provided by Università degli Studi di Cagliari within the CRUI-CARE Agreement.

Open Access This article is licensed under a Creative Commons Attribution 4.0 International License, which permits use, sharing, adaptation, distribution and reproduction in any medium or format, as long as you give appropriate credit to the original author(s) and the source, provide a link to the Creative Commons licence, and indicate if changes were made. The images or other third party material in this article are included in the article's Creative Commons licence, unless indicated otherwise in a credit line to the material. If material is not included in the article's Creative Commons licence and your intended use is not permitted by statutory regulation or exceeds the permitted use, you will need to obtain permission directly from the copyright holder. To view a copy of this licence, visit http://creativecommons.org/licenses/by/4.0/.

\section{Appendix A: A short summary of Orlicz spaces}

Let $\Phi$ be a continuous function which is in addition convex, even, satisfying the conditions $\Phi(0)=0, \Phi(-x)=\Phi(x) \geqslant 0$ for every $x \in \mathbb{R}$ and $\lim _{x \rightarrow \infty} \Phi(x)=\infty$. We call $\Phi$ a Young function. The conjugate function of $\Phi$ is defined as:

$$
\Psi(y):=\sup _{x \geqslant 0}\{x y-\Phi(x)\}, \quad \text { for every } y \geqslant 0 .
$$


For any Young function $\Phi$, let $L^{\Phi}$ be the linear space

$$
\left\{X \in L^{0}(\Omega, \mathscr{F}, \mathrm{P}) \mid \mathrm{EP}_{\mathrm{P}}(c X)<\infty, \text { for some } c>0\right\},
$$

called Orlicz Space; the expectation $\mathrm{E}_{\mathrm{p}}$ is taken with respect to the probability measure P. Any $L^{\Phi}$ admits two equivalent norms: A Luxemburg Norm, given by

$$
\|X\|_{\Phi}=\inf \left\{\lambda>0 \mid \mathrm{E}_{\mathrm{P}}\left(\Phi\left(X \lambda^{-1}\right)\right) \leqslant 1\right\}
$$

and an Orlicz Norm defined on $L^{\Psi}$ as

$$
\|X\|_{\Phi}^{*}=\sup \left\{\mathrm{E}_{\mathrm{P}}(X Y) \mid\|Y\|_{\Psi} \leqslant 1\right\}
$$

For both norms, the partial ordering $\geqslant$ in the P-a.s. sense makes $L^{\Phi}$ a Banach lattice. For an Orlicz space $L^{\Phi}$ given by the Young function $\Phi$, there exists an associated Orlicz heart $M^{\Phi}$ defined as:

$$
\left\{X \in L^{0}(\Omega, \mathscr{F}, \mathrm{P}) \mid \mathrm{E}_{\mathrm{P}}(c X)<\infty \text {, for every } c>0\right\}
$$

Thus, $\left\langle M^{\Phi}, L^{\Phi}\right\rangle$ is a dual pair of Orlicz spaces, see (Cheridito and Li 2009, Section 4.1).

Let us denote $L_{+}^{\Phi}=\left\{X \in L^{\Phi} \mid X \geqslant 0\right\}$ the positive cone of $L^{\Phi}$.

Definition 3 A Young function $\Phi$ defined on $\mathbb{R}$ and satisfying the conditions

(1) $\lim _{x \rightarrow 0} \frac{\Phi(x)}{x}=0$

(2) $\lim _{x \rightarrow \infty} \frac{\Phi(x)}{x}=\infty$

(3) $\Phi(x)=0$ implies $x=0$,

is called a $N$-Young function.

Definition 4 A Young function $\Phi$ defined on $\mathbb{R}$ satisfies the $\Delta_{2}$-property if there exists a constant $k>0$ and $x_{0} \in \mathbb{R}$ such that $\Phi(2 x) \leqslant k \Phi(x)$, for every $x \geqslant x_{0}$.

For a full treatment of Orlicz spaces see the classical Krasnoselski and Sobolev (1955) and Rao and Ren (1991). A systematic review is also contained in Edgar and Sucheston (1992).

\section{Appendix B: A short summary of ordered linear spaces}

An ordered linear space $E$ is a vector space endowed with a partial ordering relation, i.e. a binary relation denoted $\geqslant$ which is:

- reflexive, $x \geqslant x$ for every $x \in E$;

- antisymmetric, $x \geqslant y$ and $y \geqslant x$ implies $x=y$; 
- transitive, $x \geqslant y$ and $y \geqslant z$ implies $x \geqslant z$;

- compatible with the linear structure of $E$, i.e. if $x \geqslant y$ then $\lambda \cdot x \geqslant \lambda \cdot y$ for every $\lambda \in \mathbb{R}_{+}$and $x+z \geqslant y+z$, for every $z \in E$.

The positive cone of the ordered linear space is the set $E_{+}:=\{x \in E \mid x \geqslant 0\}$, where by a cone $C \subset E$ we mean a set satisfying the properties

$-C+C \subset C$

$-\lambda C \subset C$, for every $\lambda \in \mathbb{R}_{+}$;

$-C \cap(-C)=\{0\}$.

Any cone defines a partial order on $E$ as follows: $x \geqslant C y \Longleftrightarrow x-y \in C$. If $E$ is in addition a normed space, then given its topological dual $E^{*}$ we call a functional $f \in E^{*}$ a positive functional with respect to the cone $E_{+}$provided $f(x) \geqslant 0$ for every $x \in E_{+}$. If $f(x)>0$ for every $x \in E_{+} \backslash\{0\}$, then it is a strictly positive functional on the cone. A base $B \subset E_{+}$is a set containing those $b$ such that $\lambda \cdot b=x$, for every $x \in E_{+} \backslash\{0\}$ and $\lambda>0$. The base $B$ defined by some strictly positive functional $f$ is the subset $B_{f}=\left\{x \in E_{+}, \mid f(x)=1\right\}$. A bounded base $B_{f}$ corresponds to a uniformly monotonic functional $f \in E^{*}$, such that there exists some $t>0$ which yields $f(x) \geqslant t\|x\|$. For a full reference to linear spaces and the alike see Aliprantis and Border (2006).

\section{Appendix C: Useful Lemmas}

The following result complements the Fenchel-Moreau dual characterization of the penalty function used in the main robust representation result of Sect. 3.1 (Theorem $1)$.

Lemma 1 Let $\hat{\gamma}(a):=\sup \left\{\langle-X, a\rangle \mid X \in \mathcal{C}_{\rho}\right\}$. Then, $\hat{\gamma}(a)=\gamma(a)$.

Proof We have that $\gamma(a) \geqslant \hat{\gamma}(a)$, because for any $X \in \mathcal{C}_{\rho}$ it holds $\langle-X, a\rangle-$ $\rho(X) \geqslant\langle-X, a\rangle$ and then

$$
\begin{aligned}
& \sup \left\{\langle-X, a\rangle-\rho(X) \mid X \in \mathscr{R}^{\Phi}\right\} \geqslant \sup \left\{\langle-X, a\rangle-\rho(X) \mid X \in \mathcal{C}_{\rho}\right\} \\
& \quad \geqslant \sup \left\{\langle-X, a\rangle \mid X \in \mathcal{C}_{\rho}\right\} .
\end{aligned}
$$

For the reverse inequality, we take any $X \in \mathscr{R}^{\Phi}$ and set $X^{\prime}=X+\rho(X) \mathbf{1}$. We notice that $\rho\left(X^{\prime}\right)=0$, hence $X^{\prime} \in \mathcal{C}_{\rho}$ and consequently $\hat{\gamma}(a) \geqslant\langle-X, a\rangle=\langle-X, a\rangle-\rho(X)$. By taking the supremum of $\langle-X, a\rangle-\rho(X)$ all over $X \in \mathscr{R}^{\Phi}$ we get $\hat{\gamma}(a) \geqslant \gamma(a)$ for every $a \in \mathscr{B}_{1}$, and the proof is complete.

The following two lemmas are used in Corollary 2.

Lemma 2 Let $\rho_{x}(X)$ be defined as in (8), for every $x \in \mathbb{R}_{+}$and $X \in \mathscr{R}^{\Phi}$. If $\alpha$ : $\mathscr{R}^{\Phi} \rightarrow[0,+\infty]$ is an AI for processes, then $\rho_{x}(X)$ is a coherent risk measure for processes. 
Proof First, we prove (M) of Definition 1. Take any $x \in \mathbb{R}_{+}$and $X, Y \in \mathscr{R}^{\Phi}$ such that $X \geqslant Y$. By monotonicity of the AI we have

$$
\alpha(Y+m \mathbf{1}) \leqslant \alpha(X+m \mathbf{1}), \quad \text { for every } m \in \mathbb{R} \text {. }
$$

Thus, we deduce

$$
\{m \in \mathbb{R} \mid \alpha(X+m \mathbf{1}) \geqslant x\} \supset\{m \in \mathbb{R} \mid \alpha(Y+m \mathbf{1}) \geqslant x\},
$$

and taking the infimum of both sets we get

$$
\rho(X):=\inf \{m \in \mathbb{R} \mid \alpha(X+m \mathbf{1}) \geqslant x\} \leqslant\{m \in \mathbb{R} \mid \alpha(Y+m \mathbf{1}) \geqslant x\}:=\rho(Y) .
$$

To prove (P) of Definition 1, it suffices to call for the scale invariance of $\alpha$. Take $m_{1}, m_{2} \in \mathbb{R}$ such that

$$
\alpha\left(X+m_{1} \mathbf{1}\right) \geqslant x \text { and } \alpha\left(Y+m_{2} \mathbf{1}\right) \geqslant x,
$$

for every $X, Y \in \mathscr{R}^{\Phi}$ and $x \in \mathbb{R}_{+}$. By quasi-concavity of $\alpha$, for any $\lambda \in[0,1]$ we have

$$
\alpha\left(\lambda X+\lambda m_{1} \mathbf{1}+(1-\lambda) Y+(1-\lambda) m_{2} \mathbf{1}\right) \geqslant x,
$$

at the same acceptability level $x>0$. Choosing $\lambda=\frac{1}{2}$ and using again scale invariance of the AI entails

$$
\alpha\left(X+Y+\left(m_{1}+m_{2}\right) \mathbf{1}\right) \geqslant x .
$$

Therefore, the scalar $m_{1}+m_{2}$ belongs to the set $\{m \in \mathbb{R} \mid \alpha(X+Y+m \mathbf{1}) \geqslant x\}$ and it is greater than or equal to the infimum over the same set, which in turn is just $\rho(X+Y)$. This inequality holds true for all $m_{1}$ and all $m_{2}$ belonging to $\{m \in \mathbb{R} \mid \alpha(X+m \mathbf{1}) \geqslant x\}$ and to $\{m \in \mathbb{R} \mid \alpha(Y+m \mathbf{1}) \geqslant x\}$, respectively. As a consequence, taking the infimum with respect to $m_{1}$ and then $m_{2}$ we get

$$
\rho(X)+\rho(Y) \geqslant \rho(X+Y) .
$$

To prove (T) of Definition 1, we exhibit the following chain of equalities, for every $x \in \mathbb{R}_{+}$and $X \in \mathscr{R}^{\Phi}:$

$$
\begin{aligned}
\rho(X+c \mathbf{1}) & :=\inf \{m \in \mathbb{R} \mid \alpha(X+c \mathbf{1}+m \mathbf{1}) \geqslant x\} \\
& =\inf \{m \in \mathbb{R} \mid \alpha(X+(c+m) \mathbf{1}) \geqslant x\} \\
& =\inf \{c+m \in \mathbb{R} \mid \alpha(X+(c+m) \mathbf{1}) \geqslant x\}-c \\
& =\inf \{r \in \mathbb{R} \mid \alpha(X+r \mathbf{1}) \geqslant x\}-c \\
& =: \rho(X)-c .
\end{aligned}
$$


Lemma 3 Let $\left(\rho_{x}(X)\right)_{x \in \mathbb{R}_{+}}$be an increasing family of coherent risk measures for every $X \in \mathscr{R}^{\Phi}$. Then, the map $\alpha: \mathscr{R}^{\Phi} \rightarrow[0,+\infty]$ defined as

$$
\alpha(X):=\sup \left\{x \in \mathbb{R}_{+} \mid \rho_{x}(X) \leqslant 0\right\}
$$

is an AI for processes (we assume $\sup \varnothing=0$ ).

Proof Let $x>0$, then by monotonicity (M) of Definition 1 we have

$$
\rho_{x}(X) \leqslant \rho_{x}(Y), \text { for all } X, Y \in \mathscr{R}^{\Phi} \text { such that } X \geqslant Y \text {. }
$$

For any $x_{0} \in\left\{x \in \mathbb{R}_{+} \mid \rho_{x}(X) \leqslant 0\right\}$ we also have $\rho_{x_{0}}(X) \leqslant 0$, which together with (M) entails

$$
\rho_{x_{0}}(X) \leqslant \rho_{x_{0}}(Y) \leqslant 0, \text { for all } X \geqslant Y \text {. }
$$

As a consequence, the set inclusion

$$
\left\{x \in \mathbb{R}_{+} \mid \rho_{x}(X) \leqslant 0\right\} \supset\left\{x \in \mathbb{R}_{+} \mid \rho_{x}(Y) \leqslant 0\right\}
$$

holds, and taking supremum of both sides the monotonicity of AI is proved. To check quasi-concavity of $\alpha$, we first pick $X, Y \in \mathscr{R}^{\Phi}$ such that $\alpha(X) \geqslant x_{0}$ and $\alpha(Y) \geqslant x_{0}$ whenever $x_{0} \in(0,+\infty]$. By definition (12) of AI together with (M) of $\rho_{x}$ we have

$$
\rho_{x}(X) \leqslant \rho_{x_{0}}(X) \leqslant 0 \text { and } \rho_{x}(Y) \leqslant \rho_{x_{0}}(Y) \leqslant 0,
$$

for all $x<x_{0}$. This combined with property (P) of $\rho_{x}$ yields

$$
\rho_{x}(\lambda X)=\lambda \rho_{x}(X) \leqslant 0, \quad \rho_{x}((1-\lambda) Y)=(1-\lambda) \rho_{x}(Y) \leqslant 0,
$$

for every $\lambda \in[0,1]$. Moreover, by subadditivity (S) of $\rho_{x}$ and again for every $x<x_{0}$ we additionally have

$$
\rho_{x}(\lambda X+(1-\lambda) Y) \leqslant 0
$$

which entails sup $\left\{x \in \mathbb{R}_{+} \mid \rho_{x}(\lambda X+(1-\lambda) Y) \leqslant 0\right\} \geqslant x_{0}$. Eventually, this combined with definition (12) of AI yields $\alpha(\lambda X+(1-\lambda) Y) \geqslant x_{0}$ and quasi-concavity follows. Finally, scale invariance of AI follows immediately from (P) of $\rho_{x}$ in Definition 1 (positive homogeneity).

\section{Appendix D: Proofs of the main results}

Proof (Theorem 1) By the first expression of $\gamma$, we have that

$$
\rho(X) \geqslant \sup \left\{\langle-X, a\rangle-\gamma(a) \mid a \in \mathscr{B}_{1}\right\}
$$


for every $X \in \mathscr{R}^{\Phi}$. To get the desired equality, we have to prove there is no $Y \in \mathscr{R}^{\Phi}$ such that the above is a strict inequality. In fact, we can find some $m \in \mathbb{R}$ yielding

$$
\rho(Y)>m>\sup \left\{\langle-Y, a\rangle-\gamma(a) \mid a \in \mathscr{B}_{1}\right\},
$$

and consequently $\rho(Y+m \mathbf{1})>0$. This implies the singleton $\{Y+m \mathbf{1}\}$ does not belong to the acceptance set $\mathcal{C}_{\rho}$. Indeed, the latter is $\sigma\left(\mathscr{R}^{\Phi}, \mathscr{A}^{\Psi}\right)$-closed and the singleton is $\sigma\left(\mathscr{R}^{\Phi}, \mathscr{A}^{\Psi}\right)$-compact. Both sets are convex; thus, there exist some non-null $b \in \mathscr{A}^{\Psi}$, some $r \in \mathbb{R}$ and some $\epsilon>0$ such that

$$
\langle Y+m \mathbf{1}, b\rangle \geqslant r+\epsilon>r \geqslant\langle X, b\rangle, \quad \text { for all } X \in \mathcal{C}_{\rho},
$$

by the strong form of the separation theorem in locally convex spaces. The bi-linear form associated with $b$ takes negative values on $\mathcal{C}_{\rho}$. Otherwise we can find some $X_{0} \in \mathcal{C}_{\rho}$ with $\left\langle X_{0}, b\right\rangle>0$, but then the above separation inequalities are violated since in this case $\lim _{\lambda \rightarrow+\infty}\left\langle\lambda X_{0}, b\right\rangle>\langle X+\mathbf{1}, b\rangle$. Henceforth, we may assume $-b=a \in \mathscr{B}_{1}$ and then

$$
\langle Y+m \mathbf{1}, a\rangle>\sup \left\{\langle-X, a\rangle \mid X \in \mathcal{C}_{\rho}\right\}=\gamma(a) .
$$

This implies $\langle Y, a\rangle-\gamma(a)>m$ and consequently $m>\langle Y, a\rangle-\gamma(a)>m$, a contradiction. As a by product, the equality in the representation of $\rho$ must hold.

Proof (Theorem 2) Given $X \in \mathcal{C}_{\rho}$ we have $X+1 \rho(X) \in \mathcal{C}_{\rho}$. If $a \in \mathscr{B}_{1} \cap \mathcal{C}_{\rho}^{0}$ then $\langle X, a\rangle+\rho(X) \geqslant 0$, which equals $\rho(X) \geqslant\langle-X, a\rangle$. Moreover, for every $\epsilon>0$ and $X \in \mathcal{C}_{\rho}$ we have $\{X-(\rho(X)-\epsilon) \mathbf{1}\} \notin \mathcal{C}_{\rho}$. Due to the coherence of $\rho$, the acceptance set is convex and by assumption is also closed. The singleton $\{X-\epsilon \mathbf{1}\}$ is compact and convex. Therefore, there exist some non-null $a_{0} \in \mathscr{A}^{\Psi}$, some $s \in \mathbb{R}$ and $\delta>0$ such that

$$
\left\langle X, a_{0}\right\rangle \geqslant s>s+\delta \geqslant\left\langle X-(\rho(X)-\epsilon) \mathbf{1}, a_{0}\right\rangle .
$$

Moreover, due to the coherence of $\rho$ the acceptance set is a cone and we have $s=0$. This implies $\left\langle\mathbf{1}, a_{0}\right\rangle=1$ provided that $a_{0} \in \mathscr{B}_{1} \cap \mathcal{C}_{\rho}^{0}$. We finally get

$$
\sup \left\{\langle-X, a\rangle \mid a \in \mathscr{B}_{1} \cap \mathcal{C}_{\rho}^{0}\right\}+\epsilon \geqslant\left\langle-X, a_{0}\right\rangle+\epsilon \geqslant \rho(X),
$$

and since $\epsilon$ is arbitrary the representation (3) holds.

Proof (Corollary 1) By definition $f=-\rho$. This together with the dual representation (2) in Theorem 1 yield

$$
f(X)=-\sup \left\{\langle-X, a\rangle-\gamma(a) \mid a \in \mathscr{B}_{1}\right\}=\inf \left\{\langle X, a\rangle+\gamma(a) \mid a \in \mathscr{B}_{1}\right\} .
$$

Since by Lemma $1 \gamma(a)=\sup \left\{\langle-X, a\rangle \mid X \in \mathcal{C}_{\rho}\right\}$, then the penalty function equals $-\inf \left\{\langle X, a\rangle \mid X \in \mathcal{C}_{f}\right\}$. Finally, $\mathcal{C}_{f}=\mathcal{C}_{\rho}$ because $f(X) \geqslant 0 \Longleftrightarrow \rho(X) \leqslant 0$. 
Proof (Theorem 3) Either $\gamma(a)=\infty$ or $\gamma(a)<\infty$, and we have:

$$
|\sup \langle-Y, a\rangle-\sup \langle-X, a\rangle| \leqslant|\sup \langle X-Y, a\rangle| \leqslant\|X-Y\|_{\Phi}\|a\|_{\Psi},
$$

where the supremum are taken all over $a \in \mathscr{B}_{1}$ for fixed $X, Y \in \mathscr{R}^{\Phi}$. Since by assumption 1 defines a bounded base on $\mathscr{A}_{+}^{\Psi}$ the above inequalities are majorized by $c\|X-Y\|_{\Phi}$, where $c>0$. Therefore,

$$
|\rho(X)-\rho(Y)| \leqslant c\|X-Y\|_{\Phi}
$$

which implies norm and weak continuity as well.

Proof(Theorem 4) Take $a \in \mathscr{B}_{1} \cap \mathcal{C}_{\rho}^{0}$ and proceed as in the proof of Theorem 3.

Proof (Corollary 2) (a) It suffices to set $\left(\rho_{x}\right)_{x \in \mathbb{R}_{+}}$as

$$
\rho_{x}(X):=\inf \{m \in \mathbb{R} \mid \alpha(X+m \mathbf{1}) \geqslant x\}, \text { for all } x \in \mathbb{R}_{+}, \quad X \in \mathscr{R}^{\Phi} .
$$

By Lemma 2, the above is an increasing family of coherent risk measures for processes. Thus, for each acceptability level $x>0$ we define

$$
\alpha(X):=\sup \left\{x \in \mathbb{R}_{+} \mid \rho_{x}(X) \leqslant 0\right\}
$$

and note that for every $k>\alpha(X)$ we also have $\rho_{x}(X)>0$. As a consequence,

$$
\inf \{m \in \mathbb{R} \mid \alpha(X+m \mathbf{1})>k\}
$$

and moreover $\alpha(X)=\alpha(X+0 \mathbf{1})<k$ for every $k>\alpha(X)$ as defined above, and this shows that

$$
\alpha(X) \leqslant \sup \left\{x \in \mathbb{R}_{+} \mid \rho_{x}(X) \leqslant 0\right\} .
$$

Since in a similar fashion we can prove the reverse inequality, then we are done.

(b) Let $\alpha: \mathscr{R}^{\Phi} \rightarrow[0,+\infty]$ be defined by

$$
\alpha(X):=\sup \left\{x \in \mathbb{R}_{+} \mid \rho_{x}(X) \leqslant 0\right\},
$$

where $\rho_{x}$ is taken from the corresponding family of increasing coherent risk measures for processes. By Lemma 3, $\alpha$ is an AI for processes and

$$
\rho_{x}(X):=\inf \{m \in \mathbb{R} \mid \alpha(X+m \mathbf{1}) \geqslant x\}, \text { for all } x \in \mathbb{R}_{+}, \quad X \in \mathscr{R}^{\Phi} .
$$


Proof (Theorem 5) Let us assume $\alpha$ is an AI. By definition the acceptability sets form a decreasing family, i.e. $\mathscr{C}_{y}^{\alpha} \subset \mathscr{C}_{x}^{\alpha}$ whenever $x \leqslant y$ for every $x, y \in \mathbb{R}_{+}$, i.e. the acceptability sets are decreasing in $x$ for a fixed path $X \in \mathscr{R}^{\Phi}$. If we define

$$
\rho_{x}(X):=\inf \{m \in \mathbb{R} \mid \alpha(X+m \mathbf{1}) \geqslant x\}, \text { for every } x \in \mathbb{R}_{+}, X \in \mathscr{R}^{\Phi},
$$

then by Lemma 2 the map $\rho_{x}$ is a coherent risk measure for processes which is also continuous in the sense of Theorem 4 , provided that 1 is a bounded base on $\mathscr{A}_{+}^{\Psi}$. According to the same Theorem 4 , for $\rho_{x}$ there exists a representation set $G_{x} \subset$ $\mathscr{B}_{1} \cap \mathscr{C}_{\rho_{x}}^{0} \subset \mathscr{A}^{\Psi}$, where $\mathscr{C}_{\rho_{x}}^{0}:=\left\{a \in \mathscr{A}^{\Psi} \mid\langle X, a\rangle \geqslant 0\right\}$ is the polar set of $\mathscr{C}_{\rho_{x}}:=$ $\left\{X \in \mathscr{R}^{\Phi} \mid \rho_{x}(X) \leqslant 0\right\}$. As a consequence,

$$
\rho_{x}(X)=\sup _{g \in G_{x}}\langle-X, g\rangle \geqslant 0 \Longleftrightarrow \rho_{x}(X)=-\inf _{g \in G_{x}}\langle X, g\rangle \leqslant 0 .
$$

Considering a whole family $\left(\rho_{x}(X)\right)_{x \in \mathbb{R}_{+}}$, if $\mathscr{C}_{y}^{\alpha} \subset \mathscr{C}_{x}^{\alpha}$ then $G_{x} \subset G_{y}$, for every $x \leqslant y$ : the functionals representing the coherent risk measure $\rho_{x}$ produced by $\mathscr{C}_{x}^{\alpha}$ are enough to represent the coherent risk measure $\rho_{y}$ produced by $\mathscr{C}_{y}^{\alpha}$ and we are done.

Proof(Proposition 1) The dual representation in the proof of Theorem 5 becomes $\rho_{x}(X)=\sup _{\pi \in \mathscr{C}_{x}^{\alpha, 0}} \pi(-X)$ or equivalently $-\rho_{x}(X)=\inf _{\pi \in \mathscr{C}_{x}^{\alpha, 0}} \pi(X)$. Moreover, taking $X \in \mathscr{C}_{x}^{\alpha}$ yields $-\rho_{x}(X) \geqslant 0$. Assuming that $\left(\rho_{x}\right)_{x \in \mathbb{R}_{+}}$is a family of monotone, coherent risk measures for processes in $\mathscr{R}^{\Phi}$, we have that any $\rho_{x}$ is monotone with respect to the subset $\mathscr{R}_{+}^{\Phi}$ which implies $\mathscr{R}_{+}^{\Phi}=\mathscr{C}_{0}^{\alpha}$ for the corresponding polar sets. As a consequence, if $x>0$, then $\mathscr{C}_{x}^{\alpha, 0} \subset \mathscr{C}_{0}^{\alpha, 0}$. Thus, for the corresponding AI, we have

$$
\alpha(X)=\sup \left\{x \in \mathbb{R}_{+} \mid \inf _{\pi \in \mathscr{C}_{x}^{\alpha, 0}} \pi(X) \geqslant 0\right\}=\sup \left\{x \in \mathbb{R}_{+} \mid-\rho_{x}(X) \geqslant 0\right\}=\infty,
$$

which is arbitrage consistency.

\section{References}

Acciaio, B., Penner, I.: Dynamic risk measures. In Di Nunno, J., Øksendal, B. (eds.) Advanced Mathematical Methods for Finance, Chap. 1, pp. 11-44. Springer (2011)

Aliprantis, C.D., Border, K.C.: Infinite Dimensional Analysis (A Hitchhiker's Guide), 3rd edn. Springer (2006)

Acerbi, C., Tasche, D.: On the coherence of expected shortfall. J. Bank. Finance 26(7), 1487-1503 (2002)

Artzner, P., Delbaen, F., Eber, J.-M., Heath, D.: Coherent measures of risk. Math. Finance 9(3), 203-228 (1999)

Bakshi, G., Panayotov, G.: First-passage probability, jump models, and intra-horizon risk. J. Financ. Econ. 95, 20-40 (2010)

Basel Committee on Banking Supervision.: International Convergence of Capital Measurement and Capital Standars: A Revised Framework. Bank of International Settlements (2006)

Basel Committee on Banking Supervision.: Minimum Capital Requirements for MArket Risk. Bank of International Settlements (2019)

Bhattacharyya, M., Misra, N., Kodase, B.: MaxVar for non-normal and heteroskedastic returns. Quant. Finance 9(8), 925-935 (2009) 
Boudoukh, J., Richardson, M., Stanton, R., Whitelaw, R.F.: MaxVaR: long-horizon value-at-risk in a marking-to-market environment. J. Invest. Manag. 2, 14-19 (2004)

Cheridito, P., Delbaen, F., Kupper, M.: Coherent and convex monetary risk measures for bounded Càdlàg processes. Stoch. Proc. Appl. 112, 1-22 (2004)

Cheridito, P., Delbaen, F., Kupper, M.: Coherent and convex monetary risk measures for unounded Càdlàg processes. Finance Stoch. 10, 427-448 (2006)

Cheridito, P., Li, T.: Risk measures on Orlicz hearts. Math. Finance 19, 189-214 (2009)

Cherny, A., Madan, D.: New measures for performance evaluation. Rev. Financ. Stud. 22(7), 2571-2606 (2009)

Delbaen, F., Schachermayer, W.: The Mathematics of Arbitrage. Springer Finance (2006)

Delbaen, F.: Monetary Utility Functions. Osaka University CSFI Lecture Notes Series 3. Osaka University Press (2012)

Edgar, G.A., Sucheston, L.: Stopping Times and Directed Processes. Cambridge University Press (1992)

Farkas, W., Mathys, L., Vasiljević, N.: Intra-Horizon Expected Shortfall and Risk Structure in Models with Jumps. Math. Finanace 31(2), 772-823 (2021)

Goldberg, L.R., Mahmoud, O.: Drawdown: from practice to theory and back again. Math. Finan. Econ. 1(3), 275-297 (2017)

Kountzakis, C.E., Rossello, D.: Acceptability indices of performance for bounded Càdlàg processes. Stochastics 97(7), 1043-1063 (2020)

Krasnoselski, M.A., Sobolev, V.I.: On conditions of separability of Orlicz spaces. Izv. Acad. Nauk SSSR Ser. Math. 19(1), 59-68 (1955)

Rao, M.M., Ren, Z.D.: Theory of Orlicz Spaces. M.Dekker Inc, New York, Basel, Hong Kong (1991)

Rockafeller, T.R., Uryasev, S.: Conditional value-at-risk for general loss distributions. J. Bank. Finance 1(3), 275-297 (2002)

Schaefer, H.H.: Topological Vector Spaces Graduate Texts in Mathematics. Springer, New York (1999)

Publisher's Note Springer Nature remains neutral with regard to jurisdictional claims in published maps and institutional affiliations. 